\title{
Evaluation of Contrail Reduction Strategies Based on Aircraft Flight Distances
}

\author{
Neil Y. Chen* and Banavar Sridhar ${ }^{\dagger}$ \\ NASA Ames Research Center, Moffett Field, CA 94035-1000 \\ Jinhua $\mathrm{Li}^{\ddagger}$ \\ Stinger Ghaffarian Technologies, Inc., Moffett Field, CA 94035-1000 \\ Hok K. $\mathrm{Ng}^{\S}$ \\ University of California, Santa Cruz, Moffett Field, CA 94035-1000
}

\begin{abstract}
This paper evaluates a set of contrail reduction strategies based on the flight range of aircraft as contrail reduction strategies have different impacts on aircraft depending on how they plan to fly. In general, aircraft with longer flight distances cruise at the altitudes where contrails are more likely to form. The concept of the contrail frequency index is used to quantify contrail impacts. The strategy for reducing the persistent contrail formation is to minimize the contrail frequency index by altering the aircraft's cruising altitude. A user-defined factor is used to trade off between contrail reduction and extra $\mathrm{CO}_{2}$ emissions. A higher value of tradeoff factor results in more contrail reduction and extra $\mathrm{CO}_{2}$ emissions. Results show that contrail reduction strategies using various tradeoff factors behave differently from short-range flights to long-range flights. Analysis shows that short-distance flights $(<500$ miles) are the most frequent flights but contribute least to contrail reduction. Therefore these aircraft have the lowest priority when applying contrail reduction strategies. Medium-distance flights (500 to 1000 miles) have a higher priority if the goal is to achieve maximum contrail reduction in total; long-distance flights (1000 to 1500 miles) have a higher priority if the goal is to achieve maximum contrail reduction per flight. The characteristics of transcontinental flights ( $>1500$ miles) vary with different weather days so the priority of applying contrail reduction strategies to the group needs to be evaluated based on the locations of the contrail areas during any given day. For the days tested, medium-distance flights contribute up to $42.6 \%$ of the reduction among the groups during a day. The contrail frequency index per 1,000 miles for medium-distance, long-distance, and transcontinental flights can be reduced by an average of $75 \%$. The results provide a starting point for developing operational policies to reduce the impact of aviation on climate based on aircraft flight distances.
\end{abstract}

\section{Introduction}

Aircraft-induced environmental impact has drawn attention in recent years. ${ }^{1}$ The three largest emission impacts include direct emissions of greenhouse gases such as $\mathrm{CO}_{2}$, emissions of $\mathrm{NOx}$, and persistent contrails. Contrails are clouds that are visible trails of water vapor made by the exhaust of aircraft engines. Contrails form when a mixture of warm engine exhaust gases and cold ambient air reaches saturation with respect to water, forming liquid drops which quickly freeze. Contrails persist if aircraft are flying in certain atmospheric conditions. Persistent contrails reduce incoming solar radiation and outgoing thermal radiation in a way that accumulates heat. ${ }^{2}$ The global mean contrail coverage in 1992 was estimated to double by 2015, and quadruple by 2050 due to predicted increase in air traffic. ${ }^{3}$ Studies suggest that the environmental impact

\footnotetext{
*Research Aerospace Engineer, Systems Modeling and Optimization branch, MS 210-10, Member.

†Senior Scientist for Air Transportation Systems, Aviation Systems Division, MS 210-10, Fellow.

¥Senior Software Engineer, MS 210-8.

$\S$ Senior Software Engineer, University Affiliated Research Center, MS 210-8, Member.
} 
from persistent contrails are estimated to range from three to four times, ${ }^{4}$ to ten times ${ }^{5}$ larger than from aviation-induced emissions. To address minimizing environmental impacts due to contrails, methods to reduce aircraft induced persistent contrails have been proposed.

Various approaches have been proposed in the past to reduce the persistent contrail formation. The approach based on changing aircraft flight altitudes looks promising. Mannstein ${ }^{6}$ proposed a strategy to reduce the climate impact of contrails significantly by only small changes in individual flight altitude. Fichter ${ }^{7}$ showed that the global annual mean contrail coverage could be reduced by downshifting the cruise altitude. Williams ${ }^{8,9}$ proposed strategies for contrail reduction by identifying fixed and varying maximum altitude restriction policy. These restrictions generally imply more fuel burn, thus more emissions, and add congestion to the already crowded airspace at lower altitudes. Sridhar ${ }^{10}$ and $\mathrm{Chen}^{11}$ proposed contrail reduction strategies by altering an aircraft's cruising altitude in a fuel-efficient way. The strategies were designed without increasing congestion in the airspace. However, none of the above strategies take into account the range and duration of an aircraft's flight.

The objective of this paper is to evaluate contrail reduction strategies based on an aircraft's flight distance. Contrail reduction strategies have different effects on aircraft with different flight distances. In general, aircraft with longer flight distances cruise at the altitudes where contrails are more likely to form because of cold air temperatures. The concept of the contrail frequency index is used to quantify contrail formation. The strategy of reducing persistent contrail formation is to minimize the contrail frequency index by altering the aircraft's cruising altitude. A user-defined factor was applied to evaluate the tradeoff between contrail reduction and extra $\mathrm{CO}_{2}$ emissions. A higher tradeoff factor results in more contrail reduction and extra $\mathrm{CO}_{2}$ emissions. Contrail reduction strategies using different tradeoff factors behave differently for different flight distances. For this analysis, the flights during a day were divided into four groups: flights with flight distances less than 500 miles (short-distance flights), between 500 and 1,000 miles (medium-distance flights), between 1,000 and 1,500 miles (long-distance flights), and more than 1,500 miles (transcontinental flights).

The remainder of the paper is organized as follows. Section II provides descriptions of the contrail model, definition of contrail frequency index, and the contrail reduction strategies. Next, Section III shows the results and analysis of contrail reduction strategies applied to different ranges of flights. Finally, Section IV presents a summary and conclusions.

\section{Models and Strategies}

\section{II.A. Contrail Model and Contrail Frequency Index}

This paper follows the contrail models described in Ref. 11. The contrail models use atmospheric temperature and humidity data retrieved from the Rapid Updated Cycle (RUC) data, provided by the National Oceanic and Atmospheric Administration (NOAA). The horizontal resolution in RUC is 13-km with 37 vertical isobaric pressure levels ranging between 100 and 1000 millibar (mb) in $25 \mathrm{mb}$ increments. Since the vertical isobaric pressure levels do not correspond to 2,000 feet increments, linear interpolation was used to convert the RUC data to a vertical range from 26,000 feet to 44,000 feet with increments of 2,000 feet. This range is chosen because it generally is too warm for contrails to form below 26,000 feet and most commercial aircraft fly below 44,000 feet. The 2,000 feet increment is chosen is because in general same direction of flights have a vertical separation range of 2,000 feet due to the standard in Reduced Vertical Separation Minima. ${ }^{12}$ These modifications result in dividing the U.S. national airspace into a three dimensional grid with 337 elements along the latitude, 451 elements along the longitude, and 10 altitudes ranging from 26,000 feet to 44,000 feet.

Contrails form when a mixture of warm engine exhaust gases and cold ambient air reaches saturation with respect to water, forming liquid drops which quickly freeze. Contrails form in the regions of airspace that have ambient Relative Humidity with respect to Water (RHw) greater than a critical value $r_{\text {contr }}{ }^{13}$ Regions with $\mathrm{RHw}$ greater than or equal to $100 \%$ are excluded because clouds are already present. ${ }^{14}$ Contrails can persist when the environmental Relative Humidity with respect to Ice (RHi) is greater than $100 \%{ }^{15}$ In this paper, contrail favorable regions are defined as the regions of airspace that have $r_{\text {contr }} \leq R H w<100 \%$ and RHi $\geq 100 \%$.

The contrail frequency index (CFI) is used to quantify the severity of contrail activities and represents the number of aircraft in a volumetric element which meets conditions for persistent contrail formation. Air traffic in the U.S. can be mapped into the same volumetric grid as in the RUC data. The Contrail frequency index is zero for volumetric elements which do not meet the conditions for persistent contrail formation. 
Precise definitions of contrail frequency index are provided in Ref. 11.

\section{II.B. Contrail Reduction Strategies}

This paper uses the contrail reduction strategies described in Ref. 11. That strategy for reducing the persistent contrail formations is to minimize contrail frequency index by altering the aircraft's cruising altitude. Note that these altitude changes are subject to the cruise altitude limits of each aircraft. An additional constraint is added such that where an aircraft crosses a sector boundary and causes congestion, it will stay at the original cruise altitude. Additional conditions can be added to satisfy other operational procedures.

An Air Route Traffic Control Center (or Center) is divided into sectors horizontally and vertically which is monitored by an air traffic controller to maintain separation between aircraft. The number of aircraft in a sector is kept below a maximum, referred to as the Monitor Alert Parameter (MAP), to keep the controllers workload within acceptable limits. ${ }^{16}$ Therefore the MAP can be used to define the airspace capacity. The contrail reduction altitude changes will not change the sector counts unless they cross the sector boundaries. To address this operational constraints, the strategies only allow the altitude changes such that the aircraft count in a sector does not exceed the sector capacity after the altitude changes.

Consider the traffic situation at Kansas City Center at 8AM Eastern Daylight Time (EDT) on April 23, 2010. Kansas City Center has 15 high sectors and 11 super-high sectors. Among them, sector 31 has the highest sector count during the hour. Sector 31 has a lower bound of 37,000 feet and is on top of sector 28, 29 and 30, shown in Fig. 1. During the hour, altitude 35,000-36,999 feet has been identified as a high contrail area. The contrail reduction strategy suggests to increase the cruise altitude of those aircraft passing through this area by 2,000 feet. The move would reduce the contrail frequency index by 17 . Now consider if the move would cause congestion by examining the sector counts and capacities. The aircraft cruise altitude

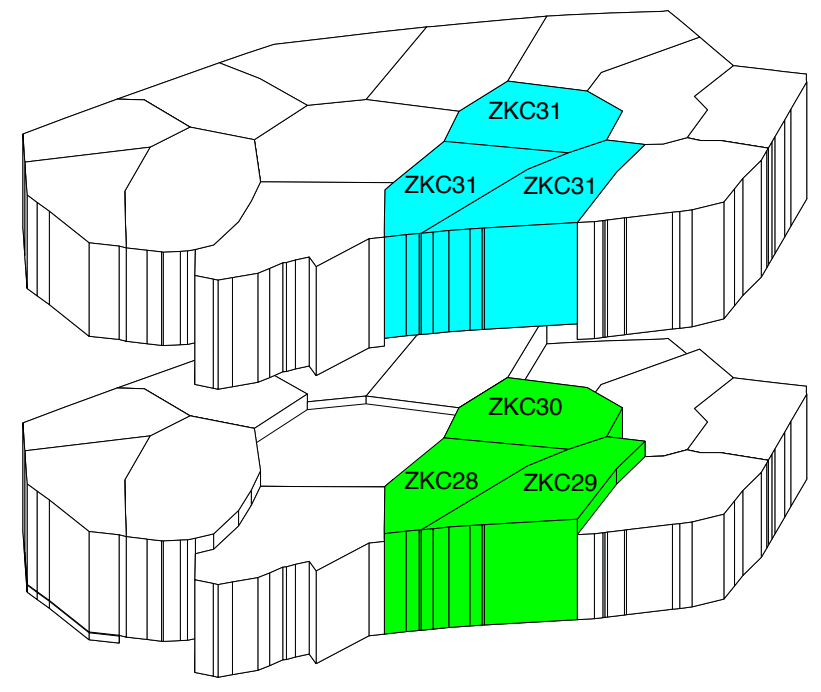

Figure 1. Kansas City Center sector 28, 29, 30 and 31.

changing from 35,000-36,999 feet to 37,000-38,999 feet would move some aircraft in sector 28,29 and 30 to sector 31. Sector 28, 29, 30 and 31 have the MAP values of 18, 18, 19 and 21 respectively. Figure 2 shows the MAP values and the sector counts in sector 28, 29, 30 and 31 before and after the altitude changes. The aircraft counts in sector 28, 29 and 30 decrease because some aircraft have been moved up to sector 31; the sector count in sector 31 increases but is still lower than the sector capacity of 21 . Thus the contrail reduction altitude changes are applied without exceeding the capacity of the airspace. In addition, contrail reduction altitude changes are only applied when the aircraft enter a new Center. The number of altitude changes is not expected to result in frequent climbs and descents to affect current operations. However, if needed, additional constraints can be imposed on the number of altitude changes.

All flights over the United States National Airspace from a 24-hour period on April 23, 2010 were analyzed. The contrail reduction strategies were applied and the results are shown in Fig. 3. The CFIs for a Center before applying the maximum reduction strategy are shown as dark blue bars. When the aircraft altitudes 

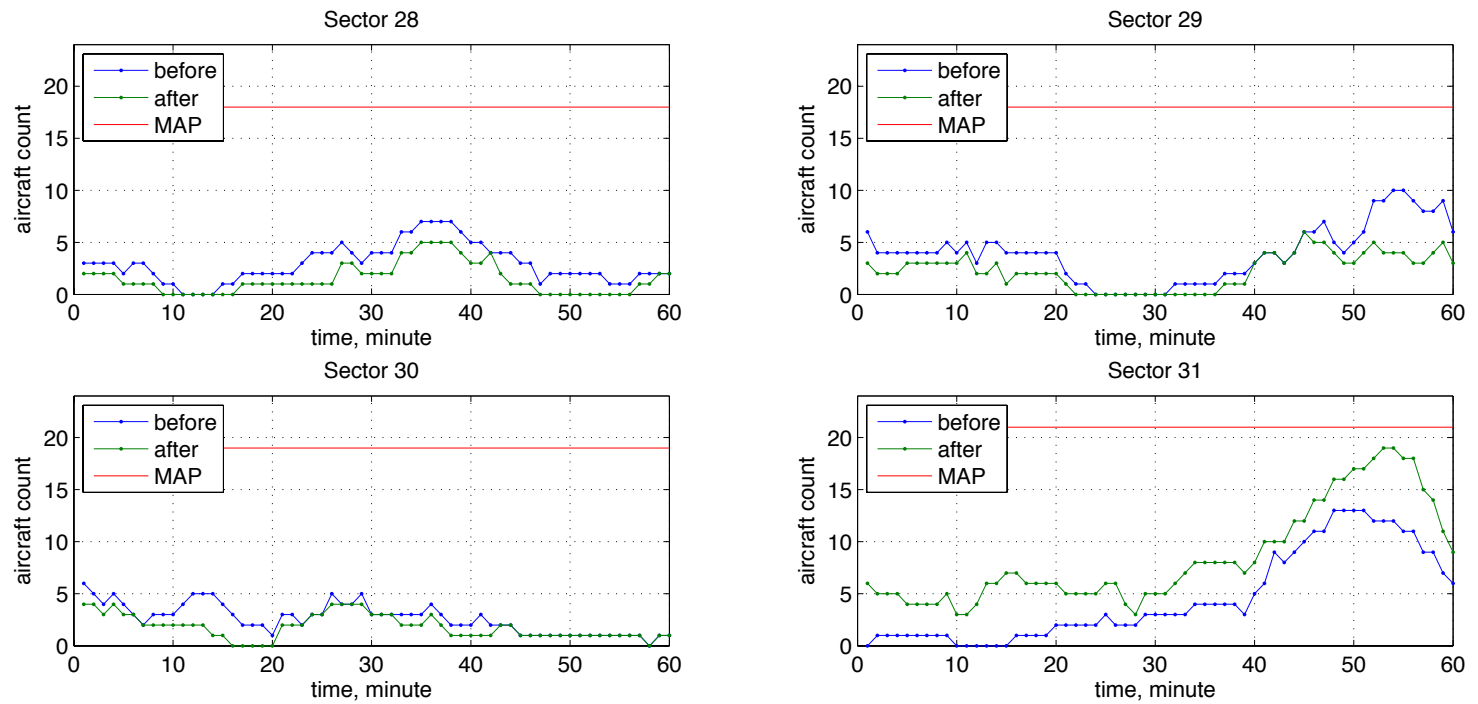

Figure 2. MAP values and sector counts before and after the contrail reduction strategies at 8AM EDT on April 23, 2010.

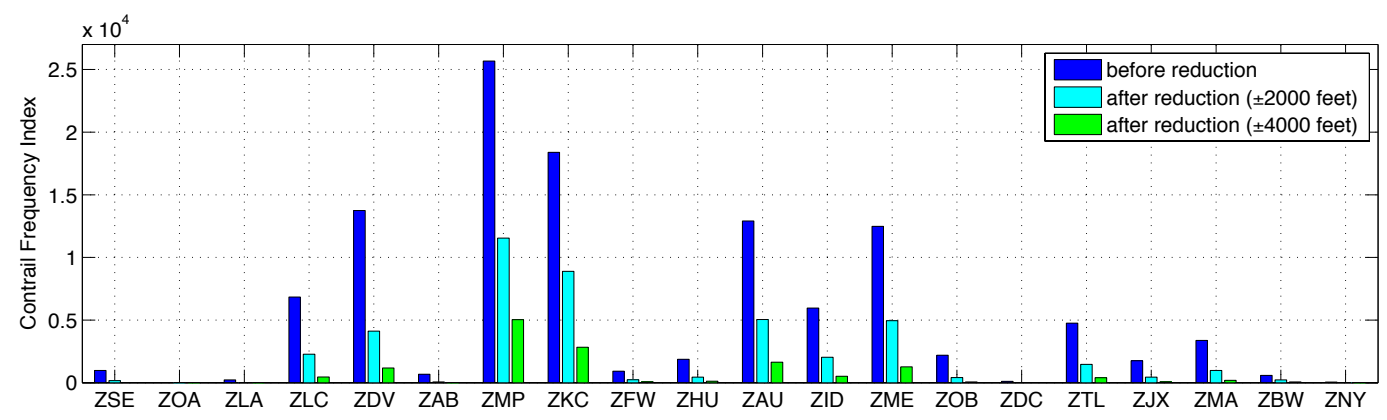

Figure 3. Results of contrail reduction strategies on April 23, 2010.

are allowed to alter by 2,000 feet, the center CFIs after reduction are shown as light blue bars. The total CFI reduction among all centers is $62 \%$. When the aircraft altitudes are allowed to alter by 4,000 feet, the total reduction is $88 \%$ as indicated as green bars. The strategies in this paper limit the altitude changes to 4,000 feet.

Altering cruising altitudes changes the aircraft fuel consumption and emissions. In order to analyze the environmental impact of contrail reduction strategies, fuel consumption and emissions are considered in the strategies. Fuel burn and emissions computations are based on a prototype of the Aviation Environmental Design Tool (AEDT) developed by the Federal Aviation Administration (FAA). ${ }^{17}$ Considering the relative environmental impact of emissions and contrails, the aircraft altitudes are modified only if the contrail reduction benefits exceed the environmental impact of additional emissions. The strategy uses a user-defined trade-off factor $\alpha$ to determine whether the strategy should apply to an aircraft. It can be interpreted as the equivalent emissions in $\mathrm{kg}$ that the user is willing to trade off for a contrail frequency index of 1 . In general, higher $\alpha$ would result in more contrail reduction and extra $\mathrm{CO}_{2}$ emissions. Figure 4 shows the amount of contrail reductions versus extra $\mathrm{CO}_{2}$ emissions using different $\alpha$ values when the aircraft altitudes are allowed to alter by 4,000 feet on April 23, 2010. In the figure, more contrail reduction takes place from left to right and more $\mathrm{CO}_{2}$ emissions occurs from bottom to top. At the lower-left point, no reduction strategy $(\alpha=0)$ is applied. The upper-right point is the maximal reduction strategy $(\alpha=\infty)$. As the value of $\alpha$ increases, the curve moves from lower-left to upper-right. The user-defined trade-off factor $\alpha$ provides a flexible way to trade off between contrail reduction and extra emissions. Better understanding of the trade-offs between contrails and emissions and impact on the climate needs to be developed to fully utilize this class of contrail reduction strategies. 


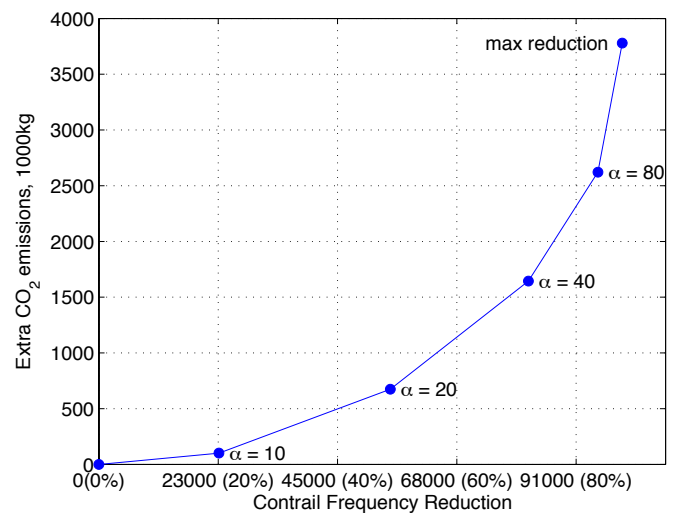

Figure 4. Contrail reduction versus extra $\mathrm{CO}_{2}$ emissions with different $\alpha$ values for all flights on April 23, 2010.

\section{Analysis}

\section{III.A. Contrail Frequency Index}

The relative climate impacts of long haul and short haul air travel were studied previously. ${ }^{18}$ For long distance flights, the fraction of the flight time spent in the high-thrust take-off and climb-out is smaller than the short distance flights. Therefore, long distance flights are more fuel efficient and generate less $\mathrm{CO}_{2}$ emissions per unit distance than short distance flights. However, short distance flights generally cruise at lower altitudes where contrails are less likely to form because the temperature is too warm. Therefore, long distance flights create more contrail impact than short distance flights. The combined climate impacts of contrails and $\mathrm{CO}_{2}$ emissions from long and short distance flights need further investigation. This section evaluates the effect of flight distances on contrail reduction strategies.

This paper defines the flight distance as the great circle distance between the origin and destination of a flight plan. The flights during a day are divided into four groups: flights with flight distance less than 500 miles (short-distance flights), between 500 and 1,000 miles (medium-distance flights), between 1,000 and 1,500 miles (long-distance flights), and more than 1,500 miles (transcontinental flights). The reason for dividing flights into such groups is to have comparable total flight distances in each group. Based on the flight data on April 23, 2010, 43\% of short-distance flights have cruise altitudes lower than 24, 000 feet, which are not likely to form contrails because of the warm temperature. On the other hand, most medium, long, and transcontinental flights cruise at high altitudes, and less than $2 \%$ of flights with flight distance more than 500 miles have cruise altitudes lower than 24,000 feet.

Data from all flights during the month of April 2010 were analyzed, and in that month, April 12, April 19 and April 3 had the highest CFI for all flights during a 24-hour period. Considering the contrail activities of different ranges of flights for these three days, the number of flights, total flight distance, total CFI and CFI per 1000 miles of range are summarized in Table 1. The group of short-distance flights has the most flights but the lowest CFI, less than $5 \%$ of the total CFI for all flights. The short-distance flights have only 0.6 to 1 CFI per flight on average, or 2.3 to 3.5 CFI per 1000 miles. Even excluding the flights with a cruise altitude lower than 24,000 feet, the average CFI per flight is 1.1 to 1.7 and the CFI per 1000 miles is 3.1 to 4.8; they are still lower than the averages in other groups. The group of transcontinental flights has the fewest flights and the greatest CFI, on April 12, among all four groups. However, on April 3 and19, its total CFIs are less than the total of groups of medium- and long- distance flights. For the month of April, transcontinental flights have the greatest CFI on 15 days. It seems that the total CFIs of transcontinental flights are more sensitive to the locations of contrail areas than the other groups, therefore the CFIs for this group has larger variance. The group of medium-distance flights has the greatest total distance. Even though its total CFI is more than in the group of long distance flights, it is consistent that its CFI per flight and CFI per 1000 miles are lower than the CFI in long-distance flights. The groups of long-distance and transcontinental flights have the grestest CFI per flight and CFI per unit distance. However, the CFI per unit distance for the group of transcontinental flights drop below the group of long and medium flights on April 19 and April 3, 2010. It is consistent that longer range of flights has more CFI per flight and, with the 
exception of the group of transcontinental flights, per unit distance.

Table 1. Summary of contrail activities for different distances of flights.

\begin{tabular}{ccccccc}
\hline \hline date & $\begin{array}{c}\text { range } \\
\text { of flights }\end{array}$ & $\begin{array}{c}\text { number } \\
\text { of flights }\end{array}$ & $\begin{array}{c}\text { total distance } \\
(1000 \text { miles })\end{array}$ & $\begin{array}{c}\text { CFI } \\
\text { (total) }\end{array}$ & $\begin{array}{c}\text { CFI } \\
\text { (per flight) }\end{array}$ & $\begin{array}{c}\text { CFI } \\
\text { (per 1000 miles) }\end{array}$ \\
\hline \multirow{4}{*}{ April 12, 2010 } & short & 13212 & 3672 & 12796 & 1 & 3.5 \\
& medium & 8096 & 5814 & 52504 & 6.5 & 9 \\
& long & 2864 & 3378 & 36021 & 12.6 & 10.7 \\
& transcontinental & 1953 & 3378 & 67420 & 34.5 & 20 \\
\hline \multirow{4}{*}{ April 19, 2010 } & short & 13365 & 3707 & 11528 & 0.9 & 3.1 \\
& medium & 8250 & 5932 & 74249 & 9 & 12.5 \\
& long & 2763 & 3257 & 54653 & 19.8 & 16.8 \\
& transcontinental & 1953 & 3399 & 40721 & 20.9 & 12 \\
\hline \hline & short & 9423 & 2637 & 6071 & 0.6 & 2.3 \\
& medium & 6592 & 4794 & 62774 & 9.5 & 13.1 \\
& long & 2687 & 3143 & 51504 & 19.2 & 16.4 \\
& transcontinental & 1705 & 3042 & 33633 & 19.7 & 11.1 \\
\hline \hline
\end{tabular}

\section{III.B. Contrail Reduction Strategies}

The contrail reduction strategies using different values of trade-off factor $\alpha$ were applied to all four groups. Figure 5 shows the amount of contrail reductions versus extra $\mathrm{CO}_{2}$ emissions using different $\alpha$ values for different flight ranges on April 3, 12 and 19,2010. The strategies limit the aircraft cruise altitude changes to 4,000 feet. In the figures, different colors indicate groups of different flight ranges. Each curve has six dots showing different strategies (right to left: $\alpha=\infty, 80,40,20,10,0$, where $\alpha=\infty$ is maximum reduction and $\alpha=0$ is no reduction). For example, in Fig $5 \mathrm{a}$, the second from the right dot of the light blue curve indicates that the contrail reduction strategy applied to transcontinental flights with a trade-off factor $\alpha=80$. It reduced the CFI on April 12 by around 40,000 with extra $\mathrm{CO}_{2}$ emissions of about 1,000 metric ton (1,000 $\mathrm{kg}$ ). The group of short-distance flights has a much smaller CFI therefore the tradeoff curves (blue) in the figures are relatively short and are located at the lower left corner. The groups of medium-distance (green curves) and long-distance (red curves) flights have similar trends where the medium-distance flight is on the right because of higher CFI to be reduced. This is true for 29 days in April, with an exception on April 28. It is noticed that the locations of the curves (light blue) for the group of transcontinental flights are not consistent across the three figures. This is also true for the entire month, which suggests that the efficiency of contrail reduction strategies for the group of transcontinental flight has different characteristics than in the other groups. It can be interpreted that the transcontinental flights have longer flight distances and the contrail reduction efficiencies are sensitive to the locations of the contrail areas. Also note that in Fig. 5c, the second dot $(\alpha=10)$ from the left of the light blue curve (transcontinental flights) has a negative value of extra $\mathrm{CO}_{2}$ emissions. This indicates that the strategy found a way to reduce both contrails and $\mathrm{CO}_{2}$ emissions for the transcontinental flights on April 3, 2010.

Table 2 summarizes the amount of contrail reductions with different $\alpha$ values for all four groups. The numbers are the CFI reductions and the percentages are over total reductions during the day. The shortdistance flights contribute the least reductions, only $9.6 \%$ to $12.6 \%$ using different $\alpha$ values on April 12, 7.3\% to $8.8 \%$ on April 19, and $3.9 \%$ to $4.3 \%$ on April 3. As described in the previous paragraph, the contrail reduction efficiencies for the group of transcontinental flights are sensitive to the locations of the contrail areas, therefore, there is no obvious trend for the reduction efficiencies compared with other groups. It contributes the most reductions, $36.3 \%$, among all reductions for the maximum reduction strategy on April 12 , but the reduction rates decay with smaller $\alpha$ values. Also, for April 3 and 19, the reduction rates are smaller than the groups of medium- and long- distance flights. Among the groups of short-, medium-, and long-distance flights, it is consistent that the group of medium flights contributes the most reduction rate, with $33.3 \%$ on April 12, 39.9\% on April 19, and $39.5 \%$ on April 3 for the maximum reduction strategies. 


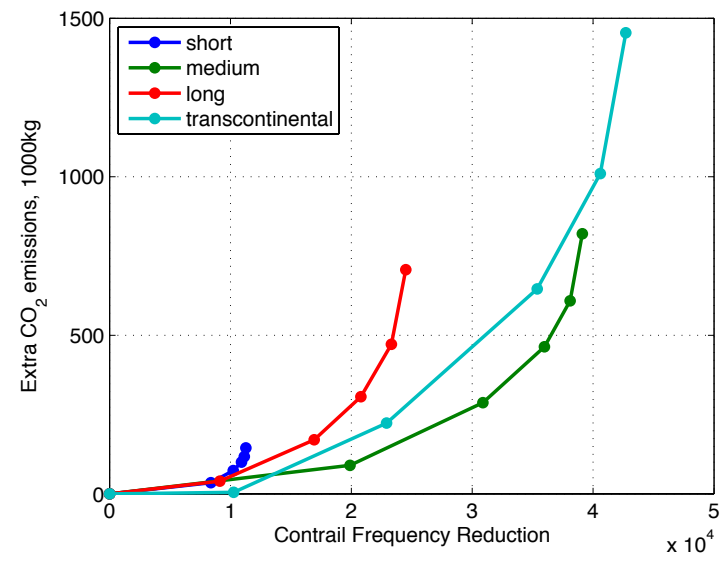

(a) April 12, 2010

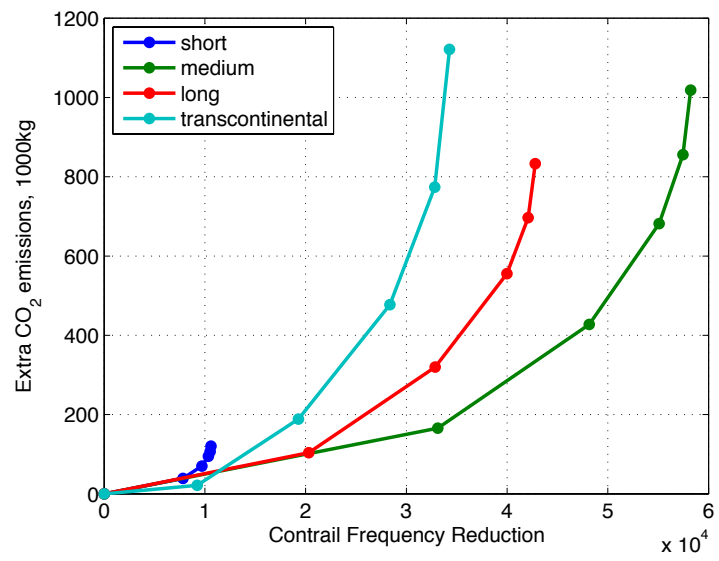

(b) April 19, 2010

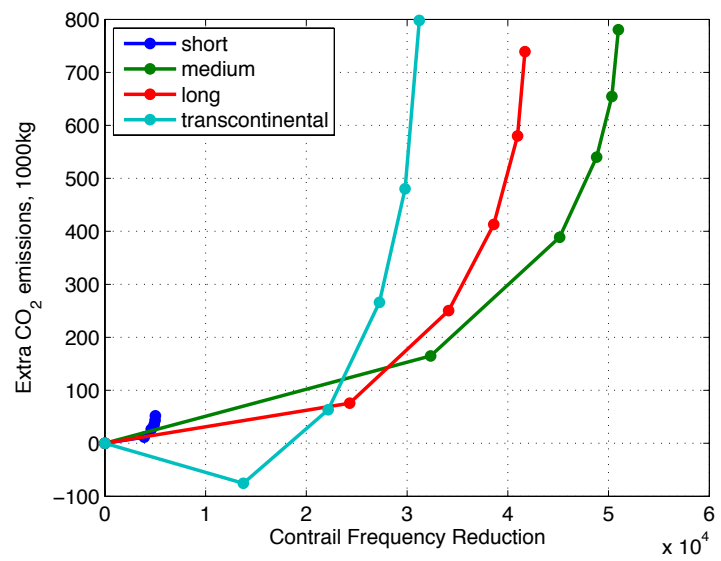

(c) April 3, 2010

Figure 5. Contrail reduction versus extra $\mathrm{CO}_{2}$ emissions with different $\alpha$ values on April 12, April 19 and April 3, 2010.

It is noticed that the contribution rates of medium-distance flights increase with smaller $\alpha$ values (smaller $\alpha$ value means less $\mathrm{CO}_{2}$ emissions). For $\alpha=10$, the reduction rate increases to $38.1 \%$ on April $12,43.8 \%$ on April 19, and $42.6 \%$ on April 3 for the maximum reduction strategies. The strategies are more efficient for transcontinental flights with larger $\alpha$ values and more efficient for short- and medium- distance flights with smaller $\alpha$ values. The percentages of the reductions are similar with different $\alpha$ values for long-distance flights.

Table 3 summarizes the CFI per 1,000 miles after the contrail reduction strategies were applied. The CFI per 1,000 miles for medium, long, and transcontinental flights can be reduced, from 9.0, 10.7 and 20.0 to 2.4, 3.5 and 8.1, respectively, on April 12; from 12.5, 16.8 and 12.0 to 2.5, 4.1 and 2.6, respectively, on April 19; from 13.1, 16.4 and 11.1 to 2.5, 3.5 and 1.6, respectively, on April 3, an average reduction of $75 \%$. The reduction rates of CFI per 1,000 miles in medium-distance flights are larger than the rates in long-distance flights but the absolute reduction values are smaller. The contrail reduction performance for the group of transcontinental flights varies with the days selected. There is no obvious trend compared with other groups. 
Table 2. Results of contrail reduction in CFIs with different $\alpha$ values.

\begin{tabular}{ccccc}
\hline \hline date & range of flights & max reduction & $\alpha=40$ & $\alpha=10$ \\
\hline \multirow{4}{*}{ April 12, 2010 } & short & $9.6 \%$ & $10.6 \%$ & $12.6 \%$ \\
& medium & $33.3 \%$ & $34.9 \%$ & $38.1 \%$ \\
& long & $20.8 \%$ & $20.2 \%$ & $20.9 \%$ \\
& transcontinental & $36.3 \%$ & $34.3 \%$ & $28.3 \%$ \\
\hline \multirow{3}{*}{ April 19,2010 } & short & $7.3 \%$ & $7.7 \%$ & $8.8 \%$ \\
& medium & $39.9 \%$ & $41.2 \%$ & $43.8 \%$ \\
& long & $29.3 \%$ & $29.9 \%$ & $29.9 \%$ \\
& transcontinental & $23.5 \%$ & $21.2 \%$ & $17.5 \%$ \\
\hline \multirow{4}{*}{ April 3,2010 } & short & $3.9 \%$ & $4.1 \%$ & $4.3 \%$ \\
& medium & $39.5 \%$ & $40.8 \%$ & $42.6 \%$ \\
& long & $32.3 \%$ & $32.3 \%$ & $32.2 \%$ \\
& transcontinental & $24.2 \%$ & $22.8 \%$ & $20.9 \%$ \\
\hline
\end{tabular}

Table 3. Contrail frequency index per 1000 miles after reduction with different $\alpha$ values.

\begin{tabular}{cccccc}
\hline \hline date & range of flights & no reduction & $\alpha=10$ & $\alpha=40$ & max reduction \\
\hline \multirow{4}{*}{ April 12, 2010 } & short & 3.5 & 1.2 & 0.5 & 0.4 \\
& medium & 9.0 & 5.7 & 3 & 2.4 \\
& long & 10.7 & 8.1 & 4.6 & 3.5 \\
& transcontinental & 20.7 & 17.7 & 10.2 & 8.1 \\
\hline \multirow{3}{*}{ April 19, 2010 } & short & 3.1 & 1 & 0.4 & 0.3 \\
& medium & 12.5 & 6.7 & 3 & 2.5 \\
& long & 16.8 & 11 & 5 & 4.1 \\
& transcontinental & 12.0 & 9.9 & 4.3 & 2.6 \\
\hline \multirow{3}{*}{ April 3, 2010 } & short & 2.3 & 0.7 & 0.3 & 0.2 \\
& medium & 13.1 & 6.4 & 2.9 & 2.5 \\
& long & 16.4 & 9 & 4.4 & 3.5 \\
& transcontinental & 11.1 & 7.4 & 2.9 & 1.6 \\
\hline \hline
\end{tabular}




\section{Conclusions}

Different concepts of contrail reduction strategies based on range of flights have been analyzed and evaluated. The concept of the contrail frequency index is used to quantify the contrail formations. The proposed strategy for reducing the persistent contrail formations is to minimize the contrail frequency index by altering the aircraft's cruising altitude within 4,000 feet. A user-defined tradeoff factor was used to trade off between contrail reductions and extra $\mathrm{CO}_{2}$ emissions. A high value of tradeoff factor results in more contrail reduction but more $\mathrm{CO}_{2}$ emissions. The results from an analysis of a month of data show that the groups of short distance flights $(<500$ miles) contributes the least to contrail reduction when the strategy is applied. The contrail reduction performance for the group of transcontinental flights ( $>1500$ miles) varies with the days selected. Among the groups of short-distance, medium-distance (500 to 1000 miles), and long-distance (1000 to 1500 miles) flights, when the strategy is applied, it is consistent that the group of medium-distance flights contributes the most contrail reduction during a day. The strategies are more efficient for transcontinental flights with larger $\alpha$ values and more efficient for short- and medium- distance flights with smaller $\alpha$ values. The percentages of the reductions are similar with different $\alpha$ values for longdistance flights. For the top three contrail days in April, 2010, the contrail frequency index per 1,000 miles for medium-range, long-range, and transcontinental flights can be reduced by an average of $75 \%$. In general, the short-distance flights are more frequent but contribute least to contrail reduction, therefore the group has the lowest priority when applying the contrail reduction strategy. The group of medium-distance flights has a higher priority if the goal is to achieve maximum contrail reduction, the group of long-distance flights has a higher priority if the goal is to achieve maximum contrail reduction per flight. The characteristics of the group of transcontinental flights vary with weather so the priority of the group needs to be further evaluated based on the locations of the contrail areas during the day. The results provide a starting point for developing operational policies to reduce the impact of aviation on climate based on aircraft flight distances.

\section{References}

\footnotetext{
${ }^{1}$ Waitz, I., Townsend, J., Cutcher-Gershenfeld, J., Greitzer, E., and Kerrebrock, J., "Report to the United States Congress: Aviation and the Environment, A National Vision, Framework for Goals and Recommended Actions," Tech. rep., Partnership for AiR Transportation Noise and Emissions Reduction, London, UK, December 2004.

${ }^{2}$ Meerkotter, R., Schumann, U., Doelling, D. R., Minnis, P., Nakajima, T., and Tsushima, Y., "Radiative forcing by contrails," Annales Geophysicae, Vol. 17, 1999, pp. 1080-1094.

${ }^{3}$ Marquart, S., Ponater, M., Mager, F., and Sausen, R., "Future Development of Contrail Cover, Optical Depth, and Radiative Forcing: Impacts of Increasing Air Traffic and Climate Change," Journal of Climate, Vol. 16, September 2003, pp. 2890-2904.

4 "The Environmental Effects of Civil Aircraft in Flight," Tech. rep., Royal Commission on Environmental Pollution, London, UK, 2002.

${ }^{5}$ Mannstein, H. and Schumann, U., "Aircraft induced contrail cirrus over Europe," Meteorologische Zeitschrift, Vol. 14, No. 4, 2005, pp. 549-554.

${ }^{6}$ Mannstein, H., Spichtinger, P., and Gierens, K., "A note on how to avoid contrail cirrus," Transportation Research. Part D, Transport and environment, Vol. 10, No. 5, September 2005, pp. 421-426.

${ }^{7}$ Fichter, C., Marquart, S., Sausen, R., and Lee, D. S., "The impact of cruise altitude on contrails and related radiative forcing," Meteorologische Zeitschrift, Vol. 14, No. 4, August 2005, pp. 563-572.

${ }^{8}$ Williams, V., Noland, R. B., and Toumi, R., "Reducing the climate change impacts of aviation by restricting cruise altitudes," Transportation Research. Part D, Transport and environment, Vol. 7, No. 6, November 2002, pp. 451-464.

${ }^{9}$ Williams, V. and Noland, R. B., "Variability of contrail formation conditions and the implications for policies to reduce the climate impacts of aviation," Transportation Research. Part D, Transport and environment, Vol. 10, No. 4, July 2005, pp. 269-280.

${ }^{10}$ Sridhar, B., Chen, N. Y., and Ng, H. K., "Fuel Efficient Strategies for Reducing Contrail Formations in United State National Air Space," 29th Digital Avionics Systems Conference, Salt Lake City, UT, October 2010.

${ }^{11}$ Chen, N. Y., Sridhar, B., and Ng, H. K., "Tradeoff between Contrail Reduction and Emissions in United States National Airspace," Journal of Aircraft, 2012, accepted.

${ }^{12}$ Schumann, U., "On Conditions for Contrail Formation from Aircraft Exhausts," Meteorologische Zeitschrift, Vol. 5, No. 1, 1996, pp. 4-23.

${ }^{13}$ Ponater, M., Marquart, S., and Sausen, R., "Contrails in a Comprehensive Global Climate Model: Parameterization and Radiative Forcing Results," Journal of Geophysical Research, Vol. 107, No. D13, 2002, pp. ACL 2-1.

${ }^{14}$ Gierens, K. M., Schumann, U., Smit, H. G. J., Helten, M., and Zangl1, G., "Determination of humidity and temperature fluctuations based on MOZAIC data and parametrisation of persistent contrail coverage for general circulation models," Annales Geophysicae, Vol. 15, 1997, pp. 1057-1066.

${ }^{15}$ Duda, D. P., Minnis, P., Costulis, P. K., and Palikonda, R., "CONUS Contrail Frequency Estimated from RUC and Flight
} 
Track Data," European Conference on Aviation, Atmosphere, and Climate, Friedrichshafen at Lake Constance, Germany, JuneJuly 2003.

${ }^{16}$ Sridhar, B., Kulkarni, D., and Sheth, K., "Impact of Uncertainty on the Prediction of Airspace Complexity of Congested Sectors," Air Traffic Control Quarterly, Vol. 19, No. 1, 2011, pp. 1-23.

${ }^{17}$ Federal Aviation Administration, Washington, DC, Aviation Environmental Design Tool (AEDT) User Guide: Beta1c, October 2010.

${ }^{18}$ Williams, V. and Noland, R. B., "Comparing the CO2 emissions and contrail formation from short and long haul air traffic routes from London Heathrow," Environmental Science 83 Policy, Vol. 9, No. 5, June 2006, pp. 487-495.

\section{0 of 10}

